\title{
Decompositions of complete graphs into bipartite 2-regular subgraphs
}

\author{
Darryn Bryant* \\ Department of Mathematics \\ University of Queensland \\ QLD 4072, Australia \\ db@maths . uq.edu . au
}

\author{
Andrea Burgess ${ }^{\dagger}$ \\ Department of Mathematical Sciences \\ University of New Brunswick \\ 100 Tucker Park Rd. P.O. Box 5050 \\ Saint John, New Brunswick \\ E2L 4L5, Canada \\ andrea.burgess@unb.ca
}

\author{
Peter Danziger $\ddagger$ \\ Department of Mathematics \\ Ryerson University \\ Toronto, Ontario \\ M5B 2K3, Canada \\ danziger@ryerson.ca
}

Submitted: Aug 26, 2014; Accepted: Mar 11, 2016; Published: Apr 1, 2016

Mathematics Subject Classifications: 05C51, 05C70, 05B30

\begin{abstract}
It is shown that if $G$ is any bipartite 2-regular graph of order at most $\frac{n}{2}$ or at least $n-2$, then the obvious necessary conditions are sufficient for the existence of a decomposition of the complete graph of order $n$ into a perfect matching and edge-disjoint copies of $G$.
\end{abstract}

\section{Introduction}

A decomposition of a graph $K$ is a set $\left\{G_{1}, G_{2}, \ldots, G_{t}\right\}$ of subgraphs of $K$ such that $E\left(G_{1}\right) \cup E\left(G_{2}\right) \cup \cdots \cup E\left(G_{t}\right)=E(K)$ and $E\left(G_{i}\right) \cap E\left(G_{j}\right)=\varnothing$ for $1 \leqslant i<j \leqslant t$. If $G$ is a fixed graph and $\mathcal{D}=\left\{G_{1}, G_{2}, \ldots, G_{t}\right\}$ is a decomposition such that $G_{i}$ is isomorphic

\footnotetext{
*Supported by the Australian Research Council via grants DP120103067, DP120100790, DP150100506, DP150100530.

†Supported by NSERC Canada through the Discovery Grant program.

$\ddagger$ Supported by NSERC Canada through the Discovery Grant program.
} 
to $G$ for $i=1,2, \ldots, t$, then $\mathcal{D}$ is called a $G$-decomposition. See [9] for a survey on $G$-decompositions, and see $[5,21]$ for general asymptotic existence results.

This paper concerns $G$-decompositions of complete graphs in the case where $G$ is a 2-regular graph. See [1] for a survey of results on $G$-decompositions of complete graphs. The complete graph of order $n$ is denoted by $K_{n}$, the cycle of order $n$ is denoted by $C_{n}$, and the path of order $n$ is denoted by $P_{n}$ (so $P_{n}$ has $n-1$ edges). If $G$ is 2-regular and $n$ is even, then there is no $G$-decomposition of $K_{n}$, and it is common to instead consider decompositions of $K_{n}-I$, where $K_{n}-I$ denotes the graph obtained from $K_{n}$ by deleting the edges of a perfect matching. For each positive integer $n$, define $K_{n}^{*}$ to be $K_{n}$ if $n$ is odd and $K_{n}-I$ if $n$ is even. The number of edges in $K_{n}^{*}$ is given by $n\left\lfloor\frac{n-1}{2}\right\rfloor$.

If $G$ is a 2-regular graph of order $k$ and there exists a $G$-decomposition of $K_{n}^{*}(n \geqslant 3)$, then it is obvious that

$$
3 \leqslant k \leqslant n \quad \text { and } \quad k \text { divides } n\left\lfloor\frac{n-1}{2}\right\rfloor .
$$

If $G$ is a 2-regular graph of order $k$, then the conditions given in (1) are called the obvious necessary conditions for the existence of a $G$-decomposition of $K_{n}^{*}$. The following problem presents itself.

Problem 1: For each 2-regular graph $G$ and each positive integer $n$ satisfying the obvious necessary conditions, determine whether there exists a $G$-decomposition of $K_{n}^{*}$.

It is known that if $G$ is a cycle, then the obvious necessary conditions are sufficient for the existence of a $G$-decomposition of $K_{n}^{*}[4,18]$. However, when $G$ is 2-regular but is not a cycle, there are cases where the obvious necessary condition are satisfied but no $G$-decomposition of $K_{n}^{*}$ exists. There is no $G$-decomposition of $K_{n}^{*}$ in each of the following cases (see [9] and [10]).

$$
\begin{gathered}
G=C_{3} \cup C_{3} \text { and } n=6, \quad G=C_{3} \cup C_{3} \text { and } n=9, \quad G=C_{4} \cup C_{5} \text { and } n=9, \\
G=C_{3} \cup C_{3} \cup C_{5} \text { and } n=11, \quad G=C_{3} \cup C_{3} \cup C_{3} \cup C_{3} \text { and } n=12 .
\end{gathered}
$$

If $G$ has order $n$, then Problem 1 is precisely the well-known Oberwolfach Problem. See $[10,11,20]$ for more information on the Oberwolfach Problem, and see [12] for a generalisation of the problem.

Problem 1 has been solved for every 2-regular graph of order at most 10 when $n$ is odd [2], and various results on Problem 1 have been obtained via graph labellings. For example, in [3] it is shown that if $G$ has order $k$ and is 2-regular with at most three components, then there exists a $G$-decomposition of $K_{2 k+1}$, and in [6] it is shown that if $G$ is bipartite and 2-regular of order $k$, then there exists a $G$-decomposition of $K_{2 k x+1}$ for each positive integer $x$. Several strong results have also been obtained on Problem 1 for the case where $G$ consists of disjoint 3-cycles [13, 14]. These results relate to Kirkman signal sets which are are used in devising codes for unipolar communication, see [15].

In [16], a simple but powerful idea is used to show that if both $n$ and $n\left\lfloor\frac{n-1}{2}\right\rfloor / k$ are even, then there is a $G$-decomposition of $K_{n}^{*}$ for every bipartite 2-regular graph $G$ of order $k$. Our main result, see Theorem 10, extends this result to the case $n\left\lfloor\frac{n-1}{2}\right\rfloor / k$ is 
odd, except when $\frac{n}{2}<k<n-2$. The special case of this extension where $k=n$ (that is, the case corresponding to the Oberwolfach Problem) is the main result in [8].

\section{Notation and Preliminary Results}

For a given graph $K$, we define the graph $K^{(2)}$ by $V\left(K^{(2)}\right)=V(K) \times \mathbb{Z}_{2}$ and $E\left(K^{(2)}\right)=$ $\left\{\{(x, a),(y, b)\}:\{x, y\} \in E(K), a, b \in \mathbb{Z}_{2}\right\}$. If $\mathcal{F}=\left\{G_{1}, G_{2}, \ldots, G_{t}\right\}$ is a set of graphs then we define $\mathcal{F}^{(2)}=\left\{G_{1}^{(2)}, G_{2}^{(2)}, \ldots, G_{t}^{(2)}\right\}$. Observe that if $\mathcal{F}$ is a decomposition of $K$, then $\mathcal{F}^{(2)}$ is a decomposition of $K^{(2)}$.

The following result of Häggkvist [16] is a critical ingredient in many of our constructions.

Theorem 1. (Häggkvist [16]) If $G$ is a bipartite 2-regular graph of order $2 m$, then there is a $G$-decomposition of $P_{m+1}^{(2)}$.

Parker [17] has completely settled the problem of decomposing complete bipartite graphs into paths of uniform length, and we need the following special case of her result.

Theorem 2. (Parker [17]) If $r$ and a are even with $r \leqslant 2 a-2, r \leqslant 2 b$, and $r$ dividing $a b$, then there is a $P_{r+1}$-decomposition of $K_{a, b}$.

We also need the following result of Tarsi on decompositions of complete graphs into isomorphic paths [19].

Theorem 3. (Tarsi [19]) There is a $P_{r+1}$-decomposition of $K_{v}$ if and only if $v \geqslant r+1$ and $r$ divides $v(v-1) / 2$.

For each even $r \geqslant 2$, let $Y_{r}$ denote any graph isomorphic to the graph with vertex set $\left\{v_{1}, v_{2}, \ldots, v_{r+1}\right\}$ and edge set

$$
\left\{v_{i} v_{i+1}: i=1,2, \ldots, r\right\} \cup\left\{v_{1} v_{3}\right\} \cup\left\{v_{i} v_{i+3}: i=2,4, \ldots, r-2\right\}
$$

$\left(E\left(Y_{2}\right)=\left\{v_{1} v_{2}, v_{2} v_{3}, v_{1} v_{3}\right\}\right)$, and let $X_{2 r}$ denote the graph obtained from $Y_{r}^{(2)}$ by adding the edges $\left\{\left(v_{1}, 0\right),\left(v_{1}, 1\right)\right\},\left\{\left(v_{2}, 0\right),\left(v_{2}, 1\right)\right\}, \ldots,\left\{\left(v_{r+1}, 0\right),\left(v_{r+1}, 1\right)\right\}$.

Lemma 4. For each even $r \geqslant 2$, there exists a decomposition of $K_{r+1}$ into $\frac{r-2}{2}$ Hamilton paths and a copy of $Y_{r}$.

Proof. Let $r \geqslant 2$ be even and for $i=0,1, \ldots, r$ let $M_{i}$ be the matching with edge set $\{\{x, y\}: x \neq y, x+y=i\}$ in the complete graph with vertex set $\mathbb{Z}_{r+1}$. Then

$$
\left\{M_{0} \cup M_{1} \cup M_{2}, M_{3} \cup M_{4}, M_{5} \cup M_{6}, \ldots, M_{r-1} \cup M_{r}\right\}
$$

is the required decomposition.

Lemma 5. If $r$ is even, $2 \leqslant r \leqslant \frac{m-1}{2}$, and $r$ divides $\frac{1}{2} m(m-1)-\frac{3 r}{2}$, then there is a $P_{r+1}$-decomposition of $K_{m}-Y_{r}$. 
Proof. By Lemma 4, there is a $P_{r+1}$-decomposition of $K_{r+1}-Y_{r}$, so it suffices to show that there is a $P_{r+1}$-decomposition of $K_{m}-K_{r+1}$. But $K_{m}-K_{r+1}$ can be decomposed into $K_{r, m-r-1}$ and $K_{m-r}$, so it suffices to prove that $K_{r, m-r-1}$ and $K_{m-r}$ each have $P_{r+1^{-}}$ decompositions. The former has a $P_{r+1}$-decomposition by Theorem 2 , and the latter by Theorem 3. It is routine to check that the hypotheses of these two theorems are satisfied when $r$ is even, $2 \leqslant r \leqslant \frac{m-1}{2}$ and $r$ divides $\frac{1}{2} m(m-1)-\frac{3 r}{2}$.

For each even $r \geqslant 2$ we define the graph $J_{2 r}$ (see Figure 1) to be the graph with vertex set

$$
V\left(J_{2 r}\right)=\left\{u_{1}, u_{2}, \ldots, u_{r+2}\right\} \cup\left\{v_{1}, v_{2}, \ldots, v_{r+2}\right\}
$$

and edge set

$$
\begin{aligned}
E\left(J_{2 r}\right)= & \left\{\left\{u_{i}, v_{i}\right\}: i=3,4, \ldots, r+2\right\} \cup \\
& \left\{\left\{u_{i}, u_{i+1}\right\},\left\{v_{i}, v_{i+1}\right\},\left\{u_{i}, v_{i+1}\right\},\left\{v_{i}, u_{i+1}\right\}: i=2,3, \ldots, r+1\right\} \cup \\
& \left\{\left\{u_{i}, u_{i+3}\right\},\left\{v_{i}, v_{i+3}\right\}\left\{u_{i}, v_{i+3}\right\},\left\{v_{i}, u_{i+3}\right\}: i=1,3, \ldots, r-1\right\} .
\end{aligned}
$$

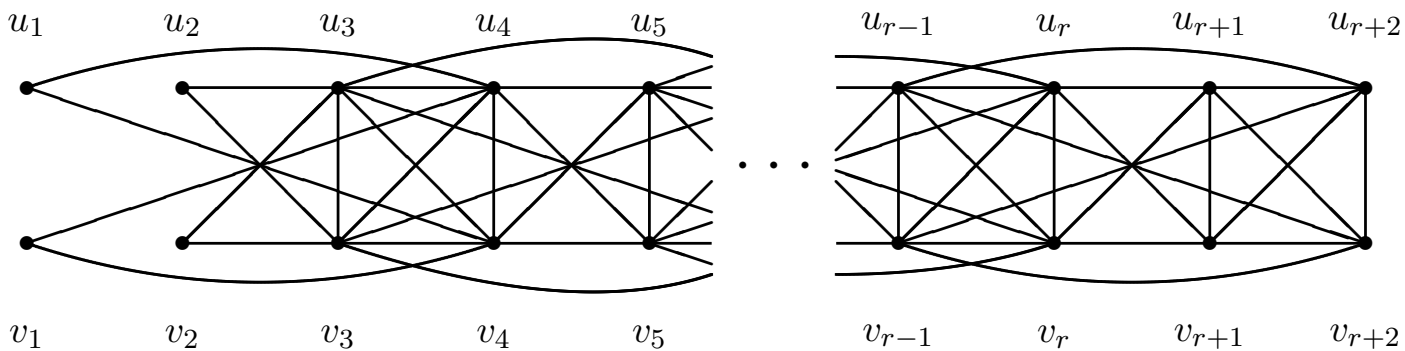

Figure 1: The graph $J_{2 r}$

The following result is proved in [8], see Lemma 10 and the proof of Lemma 11.

Lemma 6. If $G$ is a bipartite 2-regular graph of order $2 r$ where $r \geqslant 4$ is even, then there is a decomposition $\left\{\mathrm{H}_{1}, \mathrm{H}_{2}, \mathrm{H}_{3}, \mathrm{H}_{4}\right\}$ of $\mathrm{J}_{2 \mathrm{r}}$ such that

(1) $V\left(H_{1}\right)=\left\{u_{1}, u_{2}, \ldots, u_{r}\right\} \cup\left\{v_{3}, v_{4}, \ldots, v_{r+2}\right\}$,

(2) $V\left(H_{2}\right)=\left\{u_{3}, u_{4}, \ldots, u_{r+2}\right\} \cup\left\{v_{1}, v_{2}, \ldots, v_{r}\right\}$,

(3) $V\left(H_{3}\right)=\left\{u_{3}, u_{4}, \ldots, u_{r+2}\right\} \cup\left\{v_{3}, v_{4}, \ldots, v_{r+2}\right\}$,

(4) $V\left(H_{4}\right)=\left\{u_{3}, u_{4}, \ldots, u_{r+2}\right\} \cup\left\{v_{3}, v_{4}, \ldots, v_{r+2}\right\}$,

(5) each of $H_{1}, H_{2}$ and $H_{3}$ is isomorphic to $G$,

(6) $\mathrm{H}_{4}$ is a 1-regular graph of order $2 r$.

Lemma 7. If $r \geqslant 2$ is even and $G$ is any bipartite 2-regular graph of order $2 r$, then there is a decomposition of $X_{2 r}$ into three copies of $G$ and a 1-factor. 
Proof. If $r=2$, then $G$ is a 4-cycle, $X_{2 r}$ is isomorphic to $K_{6}$ and the result holds. So assume $r \geqslant 4$. Observe that if the edges $\left\{u_{1}, u_{4}\right\},\left\{u_{1}, v_{4}\right\},\left\{v_{1}, u_{4}\right\},\left\{v_{1}, v_{4}\right\}$ of $J_{2 r}$ are replaced with $\left\{u_{2}, u_{4}\right\},\left\{u_{2}, v_{4}\right\},\left\{v_{2}, u_{4}\right\},\left\{v_{2}, v_{4}\right\}$, the vertices $u_{1}$ and $v_{1}$ are deleted, and the edge $\left\{u_{2}, v_{2}\right\}$ is added, then the resulting graph is $X_{2 r}$. Let $\left\{H_{1}, H_{2}, H_{3}, H_{4}\right\}$ be the decomposition of $J_{2 r}$ given by Lemma 6 , let $H_{1}^{\prime}$ be the graph obtained from $H_{1}$ by replacing the edges $\left\{u_{1}, u_{4}\right\}$ and $\left\{u_{1}, v_{4}\right\}$ with $\left\{v_{2}, u_{4}\right\}$ and $\left\{v_{2}, v_{4}\right\}$, let $H_{2}^{\prime}$ be the graph obtained from $H_{2}$ by replacing the edges $\left\{v_{1}, u_{4}\right\}$ and $\left\{v_{1}, v_{4}\right\}$ with $\left\{u_{2}, u_{4}\right\}$ and $\left\{u_{2}, v_{4}\right\}$, let $H_{3}^{\prime}=H_{3}$ and let $H_{4}^{\prime}$ be the graph obtained from $H_{4}$ by adding the edge $\left\{u_{2}, v_{2}\right\}$ (and the vertices $u_{2}$ and $u_{4}$ ). It is easy to see that $\left\{H_{1}^{\prime}, H_{2}^{\prime}, H_{3}^{\prime}, H_{4}^{\prime}\right\}$ is the required decomposition of $X_{2 r}$.

\section{Main Results}

Lemma 8. If $n \geqslant 6$ is even and $G$ is any bipartite 2 -regular graph of order $n-2$, then there is a $G$-decomposition of $K_{n}-I$.

Proof. Let $m=\frac{n}{2}$. If $m$ is even, then let $\mathcal{D}$ be a decomposition of $K_{m}$ into $\frac{m}{2}$ Hamilton paths, and if $m$ is odd, then let $\mathcal{D}$ be a decomposition of $K_{m}$ into $\frac{m-3}{2}$ Hamilton paths and a copy of $Y_{m-1}$. The first of these decompositions exists by Theorem 3 and the second exists by Lemma 4 . In either case let the vertex set of $K_{m}$ be $\mathbb{Z}_{m}$ and let $I$ be the 1-regular graph with $V(I)=\mathbb{Z}_{m} \times \mathbb{Z}_{2}$ and edge set $E(I)=\left\{(v, 0)(v, 1): v \in \mathbb{Z}_{m}\right\}$.

Thus, $\mathcal{D}^{(2)} \cup\{I\}$ is a decomposition of $K_{n}$ into $\frac{m}{2}$ copies of $P_{m}^{(2)}$ and the perfect matching $I$ when $m$ is even, and is a decomposition of $K_{n}$ into $\frac{m-3}{2}$ copies of $P_{m}^{(2)}$, one copy of $Y_{m-1}^{(2)}$, and the perfect matching $I$ when $m$ is odd. Since the union of the copy of $Y_{m-1}^{(2)}$ and $I$ is a copy of $X_{n-2}$, the result follows by Theorem 1 and Lemma 7.

Lemma 9. Let $r \geqslant 2$. If there is a $P_{r+1}$-decomposition of $K_{m}$ or if $r$ is even and there is a $P_{r+1}$-decomposition of $K_{m}-Y_{r}$, then there is a $G$-decomposition of $K_{2 m}-I$ for every bipartite 2-regular graph of order $2 r$.

Proof. Let $G$ be a bipartite 2-regular graph of order $2 r$, let the vertex set of $K_{m}$ be $\mathbb{Z}_{m}$ and let $I$ be the 1 -regular graph with $V(I)=\mathbb{Z}_{m} \times \mathbb{Z}_{2}$ and edge set $E(I)=\{(v, 0)(v, 1)$ : $\left.v \in \mathbb{Z}_{m}\right\}$.

If there is a $P_{r+1}$-decomposition $\mathcal{D}$ of $K_{m}$, then $\mathcal{D}^{(2)}$ is a $P_{r+1}^{(2)}$-decomposition of $K_{2 m}-I$. By Theorem 1, we can decompose each copy of $P_{r+1}^{(2)}$ in $\mathcal{D}^{(2)}$ into two copies of $G$, thereby obtaining a $G$-decomposition of $K_{2 m}-I$.

Thus, we can assume $r$ is even and there is a $P_{r+1}$-decomposition of $K_{m}-Y_{r}$, and hence a decomposition $\mathcal{D}$ of $K_{m}$ into one copy of $Y_{r}$ and $\left(\left(\begin{array}{c}m \\ 2\end{array}\right)-\frac{3 r}{2}\right) / r$ copies of $P_{r+1}$. It follows that $\mathcal{D}^{(2)} \cup\{I\}$ is a decomposition of $K_{2 m}$ into one copy of $Y_{r}^{(2)},\left(\left(\begin{array}{c}m \\ 2\end{array}\right)-\frac{3 r}{2}\right) / r$ copies of $P_{r+1}^{(2)}$, and a perfect matching. There are $r+1$ edges of $I$ which form a 1-regular graph on the vertex set of the copy of $Y_{r}^{(2)}$, and the union of this 1-regular graph with the copy of $Y_{r}^{(2)}$ is a copy of $X_{2 r}$. Thus, we have a decomposition of $K_{2 m}$ into one copy 
of $X_{2 r},\left(\left(\begin{array}{c}m \\ 2\end{array}\right)-\frac{3 r}{2}\right) / r$ copies of $P_{r+1}^{(2)}$, and a matching $M$ with $m-(r+1)$ edges (such that $M$ and the copy of $X_{2 r}$ are vertex-disjoint).

By Theorem 1, we can decompose each copy of $P_{r+1}^{(2)}$ in $\mathcal{D}^{(2)}$ into two copies of $G$. Let $\mathcal{D}_{P}$ be the union of all of these decompositions. By Lemma 7 , there is a decomposition $\mathcal{D}_{X} \cup\left\{M^{\prime}\right\}$ of the copy of $X_{2 r}$ where $\mathcal{D}_{X}$ contains three copies of $G$ and $M^{\prime}$ is a perfect matching in the copy of $X_{2 r}$. This means that the union of $M$ and $M^{\prime}$ is a perfect matching in $K_{2 m}$. It follows that $\mathcal{D}_{P} \cup \mathcal{D}_{X}$ is a $G$-decomposition of $K_{2 m}-I$.

Theorem 10. Let $G$ be a bipartite 2-regular graph, let $k$ be the order of $G$, and let $n \geqslant 4$ be even. There exists a $G$-decomposition of $K_{n}-I$ if and only if $3 \leqslant k \leqslant n$ and $k$ divides $\frac{n(n-2)}{2}$, except possibly when $\frac{n}{2}<k<n-2$ and $\frac{n(n-2)}{2 k}$ is odd both hold.

Proof. The conditions $3 \leqslant k \leqslant n$ and $k$ divides $\frac{n(n-2)}{2}$ are clearly necessary for the existence of a $G$-decomposition of $K_{n}-I$. The case $k=n$ is covered by the main theorem in [8] and the case $k=n-2$ is covered by Lemma 8. If $k=n-1$, then $k$ does not divide $\frac{n(n-2)}{2}$ so there is nothing to prove. Thus, it remains only to show that there is a $G$-decomposition of $K_{n}-I$ when $3 \leqslant k \leqslant \frac{n}{2}$ and $k$ divides $\frac{n(n-2)}{2}$.

Let $m=\frac{n}{2}$ and let $r=\frac{k}{2}$ (since $G$ is bipartite, $k$ is even and $r \geqslant 2$ is an integer). By Lemma 9, it suffices to show that there is a $P_{r+1}$-decomposition of $K_{m}$ or that $r$ is even and there is a $P_{r+1}$-decomposition of $K_{m}-Y_{r}$. If $2 m(m-1) / k$ is even, then $r$ divides $m(m-1) / 2$ and so by Theorem 3 , there is a $P_{r+1}$-decomposition of $K_{m}$. If $2 m(m-1) / k$ is odd, then it follows that $r$ is even, $r$ divides $\frac{1}{2} m(m-1)-\frac{3 r}{2}$, and $k \neq m$. So $r \leqslant \frac{m-1}{2}$ and there is a $P_{r+1}$-decomposition of $K_{m}-Y_{r}$ by Lemma 5 .

Lemma 9 gives a possible approach to settling the possible exceptions in Theorem 10. The missing ingredient is $P_{r+1}$-decompositions of $K_{m}-Y_{r}$ for $\frac{m}{2}<r<m-1$ where $r=\frac{k}{2}$ and $m=\frac{n}{2}$. The first few possible exceptions in Theorem 10 are $(k, n)=(12,20)$, $(20,30),(24,42),(28,44),(36,54),(40,72),(44,68),(48,80),(52,78),(56,72),(60,92)$, $(60,102)$ and $(60,110)$.

It is worth remarking that the constructions used to prove Theorem 10 can be easily generalised as follows. In the proof of Lemma 9, each copy of $P_{r+1}^{(2)}$ can be decomposed independently, resulting in decompositions of $K_{n}-I$ into 2-regular graphs which are not all isomorphic. Although each copy of $P_{r+1}^{(2)}$ produces two isomorphic 2-regular graphs in the final decomposition, and the copy of $X_{2 r}$, when it is present, produces three isomorphic 2-regular graphs in the final decomposition, this construction can produce a wide variety of different combinations of 2-regular graphs in the final decomposition.

The 2-regular graphs given by the construction of the preceding paragraph will all have the same order, namely $k=2 r$, but it is also possible to get around this constraint. Instead of using a $P_{r+1}$-decomposition of $K_{m}$ or $K_{m}-Y_{r}$, one may use a decomposition of $K_{m}$ or $K_{m}-Y_{r}$ into paths which are not necessarily all isomorphic. In [7] it is shown that the obvious necessary conditions are sufficient for the existence of a decomposition of $K_{m}$ into paths of any specified lengths. This facilitates the construction of decompositions of $K_{n}-I$ into many combinations of 2-regular graphs of many different orders. 


\section{References}

[1] P. Adams, D. Bryant and M. Buchanan, A survey on the existence of $G$-designs, $J$. Combin. Des., 16 (2008), 373-410.

[2] P. Adams, D. Bryant and H. Gavlas, Decompositions of the complete graph into small 2-regular graphs, J. Combin. Math. Combin. Comput., 43 (2002), 135-146.

[3] A. Aguado, S. I. El-Zanati, H. Hake, J. Stob and H. Yayla, On $\rho$-labeling the union of three cycles, Australas. J. Combin., 37 (2007), 155-170.

[4] B. Alspach and H. Gavlas, Cycle decompositions of $K_{n}$ and $K_{n}-I$, J. Combin. Theory Ser. B, 81 (2001), 77-99.

[5] B. Barber, D. Kühn, A. Lo, and D. Osthus, Edge-decompositions of graphs with high minimum degree, Adv. Math., 288 (2016), 337-385.

[6] A. Blinco and S. I. El-Zanati, A note on the cyclic decomposition of complete graphs into bipartite graphs, Bull. Inst. Combin. Appl., 40 (2004), 77-82.

[7] D. Bryant, Packing paths in complete graphs, J. Combin. Theory Ser. B, 100 (2010), 206-215.

[8] D. Bryant and P. Danziger, On bipartite 2-factorisations of $K_{n}-I$ and the Oberwolfach problem, J. Graph Theory, 68 (2011), 22-37.

[9] D. Bryant and S. El-Zanati, Graph decompositions, in The CRC Handbook of Combinatorial Designs, 2 nd edition (Eds. C. J. Colbourn, J. H. Dinitz), CRC Press, Boca Raton (2007), 477-486.

[10] D. Bryant and C. A. Rodger, Cycle Decompositions, in The CRC Handbook of Combinatorial Designs, 2 nd edition (Eds. C. J. Colbourn, J. H. Dinitz), CRC Press, Boca Raton (2007), 373-382.

[11] D. Bryant and V. Scharaschkin, Complete solutions to the Oberwolfach problem for an infinite set of orders, J. Combin. Theory Ser. B, 99 (2009), 904-918.

[12] S. M. Cioabă and P. J. Cameron, A graph partition problem, Amer. Math. Monthly, 122 (2015), 972-982.

[13] C. J. Colbourn, D. Horsley and C.Wang, Trails of triples in partial triple systems, Des. Codes Cryptogr., 6 (2012), 199-212.

[14] C. J. Colbourn, D. Horsley and C. Wang, Colouring Triples in Every Way: A Conjecture, Quad. Mat., 28 (2012), 257-286.

[15] C. J. Colbourn and S. Zhao, Maximum Kirkman signal sets for synchronous uni-polar multi-user communication systems, Des. Codes Cryptogr., 20 (2000), 219-227.

[16] R. Häggkvist, A lemma on cycle decompositions, Ann. Discrete Math., 27 (1985), $227-232$.

[17] C. A. Parker, Complete bipartite graph path decompositions, PhD Thesis, Auburn University, (1998). 
[18] M. Šajna, Cycle decompositions III: complete graphs and fixed length cycles, J. Combin. Des., 10 (2002), 27-78.

[19] M. Tarsi, Decomposition of a complete multigraph into simple paths: Nonbalanced handcuffed designs, J. Combin. Theory Ser. A, 34 (1983), 60-70.

[20] T. Traetta, A complete solution to the two-table Oberwolfach problems, J. Combin. Theory Ser. A, 120 (2013), 984-997.

[21] R. M. Wilson, Decompositions of complete graphs into subgraphs isomorphic to a given graph. Proceedings of the Fifth British Combinatorial Conference (Univ. Aberdeen, Aberdeen, 1975), pp. 647-659. Congressus Numerantium, No. XV, Utilitas Math., Winnipeg, Man., 1976. 